\title{
Research
}

\section{Predictors of persistent complaints after a knee injury in primary care}

\begin{abstract}
\section{Background}

Prognosis of persistent complaints after knee injury is based on secondary care populations. In a primary care setting, however, no studies have addressed this issue.

\section{Aim}

To identify possible predictors of persistent complaints 1 year after a knee injury. These predictors are important for guiding the GP's therapeutic management, and giving advice to patients about work and/or sports-related activities.
\end{abstract}

\section{Design and setting}

Primary care prospective cohort study with a 1 year follow-up period in five municipalities in the southwest region of the Netherlands.

\section{Method}

Patients who were eligible were recruited to the study by a GP research network with around 84000 patients and 40 participating GPs. A total of 134 patients (aged $18-65$ years) who consulted their GP within 5 weeks after a knee injury entered the study. Follow-up after 1 year was conducted in 122 patients. The main outcome was persistent complaints 1 year after injury; possible predictors for these complaints were obtained with a questionnaire, a physical examination, and magnetic resonance imaging (MRI), according to a standardised protocol.

\section{Results}

After 1 year, of the 122 available patients, 21 $(17 \%)$ reported persistent complaints and 101 (83\%) reported full recovery or major improvement. In this study being aged $>40$ years had a significant association $(P<0.05)$ with persistent complaints lodds ratio $8.0,95 \%$ confidence interval 2.1 to 30.5 ). Physical examination and MRI findings revealed no predictors that were associated with these complaints.

\section{Conclusion}

Being aged $>40$ years was the only determinant with a significant association with persistent complaints. As physical examination and MRI had no predictive value, they are not recommended for prognosis of persistent complaints.

\section{Keywords}

general practice; knee injury; prognosis.

\section{INTRODUCTION}

Patients consulting their GP for disorders related to a knee injury are interested in a prediction of the course of these disorders, as well as information or advice about resuming work and/or sports activities. The GP has the difficult, but indispensable, task of predicting the patient's prognosis as accurately as possible. ${ }^{1-4}$ However, in the case of a knee injury, the GP is faced with lack of evidence.

The second Dutch national survey of complaints for which patients consulted in general practice reported an incidence of traumatic knee disorders of 5.3 per 1000 patients annually. ${ }^{5}$ Studies on the natural course of ligamentous knee injuries (reviewed by Boks et $a^{(6)}$ showed that, in the case of an anterior cruciate ligament rupture, a normal anterior cruciate ligament was found on magnetic resonance imaging (MRI) in 42\% (95\% confidence interval $[\mathrm{Cl}]=28$ to 57) at 3-month follow-up; at 11-month follow-up, results showed that further repair was possible. ${ }^{7-8}$ Partial or complete posterior cruciate ligament ruptures showed regained continuity on MRI in $93 \%$ of cases $(95 \% \mathrm{Cl}=80$ to 98$)$ at 3 year follow-up.-10 Data on the prognosis of recovery after a knee injury are solely based on secondary care populations. ${ }^{11-13}$ Longterm studies show that knee injuries are an important risk factor for the development of osteoarthritis of the knee..$^{14-16}$

MRI has become a normal procedure for

HPA Wagemakers, MSc; PAJ Luijsterburg, PhD assistant professor; EM Heintjes, PhD; BW Koes, professor; SMA Bierma-Zeinstra, professor, Department of General Practice; JAN Verhaar, professor, MD, Department of Orthopaedics Erasmus Medical Center, University Medical Center, Rotterdam, the Netherlands. MY Berger, professor, MD, Department of General Practice,

University Medical Center, Groningen, the Netherlands.

Address for correspondence

Pim Luijsterburg, Department of General Practice, non-invasive diagnosis and gradation of the severity of knee injuries in secondary care. ${ }^{17,18}$ In primary care, however, the use of $\mathrm{MRI}$ in knee injuries is not yet common practice, but is slowly being introduced. It has previously been shown that patient characteristics, history taking, and physical examination have limited value in diagnosing meniscal and ligament damage; ${ }^{19-21}$ as MRI is an additional tool in diagnosing such damage, ${ }^{17,18}$ it is worthwhile to establish whether or not it has additive prognostic value in primary care.

This study ascertains whether specific determinants from history taking and physical examination in primary care have predictive value for the prognosis of persistent complaints 1 year after an acute knee injury, and whether MRI has additional predictive value for the prognosis of these persistent knee complaints.

\section{METHOD}

Design

The Department of General Practice of Erasmus Medical Center, University Medical Center, Rotterdam, conducted a large prospective cohort study on knee complaints in primary care. A total of $40 \mathrm{GPs}$ from five municipalities in the southwest region of the Netherlands asked patients with new knee complaints to participate in the general cohort study, with a follow-up of 1 year. This network represented a total

Erasmus Medical Center, University Medical Center Rotterdam, PO Box 2040, Rotterdam, the Netherlands.

E-mail: p.luijsterburgderasmusmc.nl

Submitted: 18 November 2011; Editor's response: 20 December 2011; final acceptance: 19 January 2012

\section{British Journal of General Practice}

This is the full-length article (published online 30 Jul 2012) of an abridged version published in print. Cite this article as: Br J Gen Pract 2012; DOI: 10.3399/bjgp12X653615. 


\section{How this fits in \\ Prognosis of persistent complaints after a knee injury is based on secondary care populations. In a primary care setting. however, no studies have addressed this issue. Being aged $>40$ years was found to be a predictor for persistent complaints 1 year after a knee injury, whereas physical examination and MRI findings showed no association for persistent complaints. At present, use of physical examination or MRI for prognosis of persistent complaints after knee injury is not recommended. Based on this study, only history taking shows some prognostic value and the study cannot recommend physical examination or MRI examination for prognostic use.}

patient population of around 84000 patients. Detailed information about the study design has been published previously.22

Patients with knee complaints were informed about the general cohort study during the initial consult with the GP and were invited to participate. Those aged 18-65 years with a traumatic onset of knee complaints, and who visited their GP within 5 weeks after initial injury, were also asked to participate in an additional MRI study. After registration of the patient at the research centre of the Department of General Practice, an appointment was made, informed consent was acquired, and patients completed the baseline questionnaire If patients gave consent for the additional MRI study, an appointment for the MRI was scheduled; following this MRI, a physical examination was performed. Patients with MRI contraindications - such as pregnancy, metal implants, or a pacemaker - were excluded from this MRI study.

\section{Data collection}

At baseline, the participating patients completed a self-report questionnaire. The questionnaire collected data on age, sex, socioeconomic status, history of previous knee injuries and/or surgery, present symptoms, mechanism of injury, and the level of activity during work or sport. The therapeutic management initiated by the GP at baseline was also recorded.

The severity of pain was obtained using an 11-point numeric rating scale ranging from 0 (no pain) to 10 (unbearable pain). ${ }^{23}$ The Lysholm knee score was used to provide relevant information regarding pain, swelling, instability, and functional limitations such as walking and stair climbing. ${ }^{24}$ The Lysholm score ranged from 0 (worst possible score) to 100 (excellent score). The Tegner knee function score (range: 0-10) was used to determine the level of activity in work and sports prior to the knee injury; 0: on sick leave/disability, 10 : participation in competitive sports. ${ }^{24}$ The Tegner score was obtained with the level of work' and 'sports activities' determinants from the questionnaires. In the final questionnaire, at 1 year after baseline, patients were asked to report their perceived recovery using a seven-item Likert scale categorised as 'full recovery', 'major improvement', 'minor improvement', 'about the same', 'minor deterioration', 'major deterioration', or 'worse than ever'. 22,25

Persistent complaints were defined as those in which, 1 year after the initial knee injury, patients reported 'minor improvement', 'about the same', 'minor deterioration', 'major deterioration', or 'worse than ever'. Patients' reports of either 'full recovery' or 'major improvement' were considered as signifying a clinically relevant recovery.

The MRI was scheduled 2-6 weeks after the initial trauma and used a 1.0 Tesla General Electric device. The results of the MRI were determined by two radiologists independently, based on a standardised classification form. In cases of disagreement, the findings were discussed until consensus was reached. Meniscal tears were classified as horizontal, longitudinal, radial, or complex. Anterior cruciate ligament, posterior cruciate ligament, medial cruciate ligament, and lateral collateral ligament lesions were classified as either partial or complete. At the time of data collection for this study there was no validated scoring system for osteoarthritic features seen on MRI, so the study adapted the most frequently used method - the Kellgren and Lawrence method $^{26}$ - to score the severity of osteoarthritis on X-rays. Detailed information about the MRI procedure is reported elsewhere. ${ }^{27}$

A physical examination was performed immediately after the MRI, according to a standardised protocol. ${ }^{22}$ A trained physical therapist with $\geq 15$ years experience in performing physical examination in patients with knee injuries and with $\geq 10$ years' experience in diagnostic research performed the examination. ${ }^{28}$ Physical examination of both knees consisted of inspection of alignment and joint effusion, palpation of temperature, collateral 


\section{Table 1. Characteristics of the participants at baseline and at 1-year follow-up}

\begin{tabular}{|c|c|c|}
\hline Characteristic & $\begin{array}{l}\text { Participants at baseline } \\
\qquad(n=134)\end{array}$ & $\begin{array}{l}\text { Participants at follow-up } \\
\qquad(n=122)\end{array}$ \\
\hline Age in years, mean (SD) & $40.2(12.2)$ & $40.8(12.1)$ \\
\hline Sex, female, $n(\%)$ & $70(52)$ & $54(44)$ \\
\hline BMI, mean (SD) & $26(4.3)$ & $26(4.2)$ \\
\hline SF-36 general health, mean (SD) & 76 (18) & $77(18)$ \\
\hline Symptom side right, $\mathrm{n}(\%)$ & $70(52)$ & $60(49)$ \\
\hline Pain severity, $0-10$, mean (SD) & $4.7(2.4)$ & $4.7(2.3)$ \\
\hline Lysholm knee function score, 0-100, mean (SD) & $62(22)$ & $62(22)$ \\
\hline Employed, $n(\%)$ & $113(84)$ & 89 (73) \\
\hline Sports participation, $n(\%)$ & $97(72)$ & $76(62)$ \\
\hline \multicolumn{3}{|l|}{ Cause of trauma } \\
\hline Work related, $n(\%)$ & 20 (15) & $61(46)$ \\
\hline Onset during sports activity, $n(\%)$ & $18(15)$ & $58(48)$ \\
\hline \multicolumn{3}{|l|}{ Diagnosis as seen on MRI } \\
\hline No lesion, $n(\%)$ & 52 (39) & $82(61)$ \\
\hline Any lesion, $n(\%)$ & $28(21)$ & $6(4)$ \\
\hline ACL lesion, $n(\%)$ & $35(26)$ & $8(6)$ \\
\hline PCL lesion, $n(\%)$ & 47 (35) & 45 (37) \\
\hline MCL lesion, $n(\%)$ & $77(63)$ & $26(21)$ \\
\hline LCL lesion, $n(\%)$ & $5(4)$ & $32(26)$ \\
\hline Meniscal tear, $n(\%)$ & $8(7)$ & 45 (37) \\
\hline
\end{tabular}

$A C L=$ anterior cruciate ligament. $B M I=$ body mass index. $L C L=$ lateral collateral ligament $M C L=$ medial collateral ligament. $\mathrm{MRI}=$ magnetic resonance imaging. $P C L=$ posterior cruciate ligament. $S D=$ standard deviation. SF-36 = 36-item Short Form Health Survey.
$(P<0.10)$ to eliminate redundant variables. With the results of this multivariate analysis, a prognostic model was built and the area under the curve $(A \cup C)$ of the receiver operating characteristic (ROC) curve was calculated. Complete case analysis was used.

To determine the additive predictive value of MRI for the prognosis of persistent complaints, the associated determinants from MRI $(P<0.10)$ were added in the prognostic model using the Enter method. Again, the AUC was calculated. The additive predictive value of MRI was determined by the difference in the AUC in both models.

\section{RESULTS}

\section{Study population}

During the inclusion period, 184 patients consulting their GP because of complaints caused by a knee injury were eligible to enter the MRI study; of those, 134 (73\%) patients were included in the MRI study. The 50 non-participants filled in the baseline questionnaire to enter in the general cohort study but were unwilling or unable to participate in the additional MRI study. The participants and non-participants showed no clinically significant differences at baseline with regard to age, sex, pain severity, or Lysholm knee score. ${ }^{20}$ In addition, the patients available at follow-up ( $n=122)$ showed no relevant differences compared with the patients who were initially included ( $n=134)$ (Table 1).

The baseline questionnaires were available for 134 patients. In two patients physical examination could not be performed because of plaster immobilisation at the time of the MRI. The mean age of the participants was 40 years (range $=18-64$ years) and a small majority was male $(48 \%$, Table 1). The majority of patients (84\%) had paid employment. Before the initial trauma, 97 (72\%) patients participated in sports. Knee injuries were frequently acquired during sports activities (46\%).

\section{MRI findings}

In 52 (39\%) patients no meniscal tears, or cruciate or collateral ligament lesions were seen on the baseline MRI (Table 1). In the remaining $82(61 \%)$ patients, one of the above-mentioned tears or lesions was found on the MRI; more specifically, in 47 (35\%) patients a meniscal tear was found, while $34(25 \%)$ patients showed an anterior cruciate ligament or posterior cruciate ligament lesion. Medial collateral ligament or lateral collateral ligament lesions were 
Table 2. Perceived recovery at 1-year follow-up

\begin{tabular}{|c|c|c|c|c|c|}
\hline \multirow[b]{2}{*}{ Perceived recovery } & \multicolumn{2}{|c|}{ Clinically relevant recovery, $n(\%)$} & \multicolumn{3}{|c|}{ Persistent complaints, $n(\%)$} \\
\hline & Full recovery & Major improvement & Minor improvement & About the same & Minor deterioration \\
\hline Total group $(n=122)$ & $34(28)$ & $67(55)$ & $14(11)$ & 4 (3) & $3(2)$ \\
\hline No lesion group ( $n=45)$ & 15 (33) & $22(49)$ & $5(11)$ & $2(4)$ & $1(2)$ \\
\hline Any lesion group ( $n=77)$ & $19(25)$ & $45(58)$ & 9 (12) & 2 (3) & $2(2)$ \\
\hline
\end{tabular}

found in $43(32 \%)$ patients. In total, 36\% of the patients showed a combination of these lesions on MRI.

\section{1-year follow-up}

At 1-year follow-up, a perceived recovery score was available for 122 (91\%) patients (Table 2). Of these patients, 21 (17\%) reported persistent complaints, comprising 14, who reported 'minor improvement', four who reported 'about the same', and three who reported 'minor deterioration'. None of the patients reported 'major deterioration' or 'worse than ever'. A total of 34 patients (28\%) reported 'full recovery' and 67 (55\%) reported 'major improvement'. As such, overall, 101 (83\%) patients reported a clinically relevant recovery.

In 15 patients, a total of 19 surgical interventions were performed during the 1year follow-up: one anterior cruciate ligament reconstruction and 18 arthroscopies. When excluding these patients from the analyses the findings did not change.

\section{Binary analysis}

After the binary logistic regression analysis, four determinants from history taking showed an association with persistent complaints $(P<0.10)$ :

The research network Honeur is financially supported by the health insurance companies Trias, Zilveren Kruis Achmea, and $\mathrm{OZ}$.

\section{Ethical approval}

The study protocol was approved by the medical ethics committees of Erasmus Medical Center, Rotterdam (the study centre) and Hospital Rijnmond Zuid (the MRI location).

\section{Provenance}

Freely submitted; externally peer reviewed.

\section{Competing interests}

The authors have declared no competing interests.

\section{Discuss this article}

Contribute and read comments about this article on the Discussion Forum: http://www.rcgp.org.uk/bjgp-discuss association with persistent complaints ( $P<0.05$, OR $8.0,95 \% \mathrm{Cl}=2.1$ to 30.5 , Table 4). The AUC of the ROC of this model was 0.81 .

Adding the determinants from MRI with an additive association $(P<0.10)$ to the model did not alter the results of the analysis, including the AUC of the ROC.

\section{DISCUSSION}

\section{Summary}

Of the 134 patients included in this study, 82 (61\%) showed an isolated meniscal tear, a ligament lesion, or a combination of both on the baseline MRI. In the remaining 52 (39\%) patients, none of these damages was reported.

Of the 122 patients available at 1-year follow-up, 21 (17\%) suffered from persistent complaints. The results of the multivariate analysis showed that being aged $>40$ years is the main predictor for persistent complaints 1 year after injury $(P<0.05)$. In spite of $\mathrm{ORs}$ of $\geq 2.5$, other determinants such as sex, baseline pain score, and popping sensation during trauma were not significantly associated with persistent complaints, probably due to lack of power. Physical examination showed no predictive value at all on the prognosis of persistent complaints. In addition, the MRI showed no additive prognostic value when added to the model with factors from history taking. The AUC in the model including MRI equalled the AUC only with determinants from history taking and physical examination.

During follow-up, some of the patients underwent a surgical intervention due to their knee injury. As these patients may have a different perceived recovery compared with patients without such interventions, the study performed an additional analysis excluding patients that underwent arthroscopy or surgery during the 1-year follow-up. The results of this analysis showed no difference with regard to the determinants involved.

The results suggest an influence of the presence of osteoarthritis on persistent complaints after knee injuries, although 
only $<10 \%$ showed osteoarthritis on the MRI at baseline. Further, almost all patients with persisting complaints were aged $>40$ years, which was the main predictive factor for persistent complaints and overruled the predictive value of the presence of osteoarthritis; in these patients early osteoarthritis might be due to the persistent complaints.

\section{Strengths and limitations}

To this study's knowledge, this is the first study in primary care to report on the prognosis of persistent complaints, 1 year after having sustained a knee injury. The spectrum of the damage to the knee presented to the participating GPs is wide; therefore, it can be assumed that this study population represents the population of patients with disorders caused by a knee injury consulting the GP.

In the present study, the sample was relatively limited and many variables were tested; however, all these variables are part of the normal clinical evaluation and should, in the study's opinion, be tested for their relationship with persistent complaints. For this reason, the study performed bootstrapping with 500 replications and this showed that the main predictive variable (being aged >40years) could only be replicated in 269 samples. This shows that caution is needed not to overinterpret the presented results. As such, the relationships found should preferably be replicated in other primary care cohorts. As a result of the limited sample, less prominent but related variables might have been missed, and clinically relevant subgroup analyses (for example, stratifications for age categories, or patients with any confirmed lesion on MRI, or even per lesion) could not be performed.

\section{Comparison with existing literature}

In this study's analysis, MRI features usually reported in a clinical setting were included. A range of features that might relate to early osteoarthritis were not included. For example, degenerative meniscal lesions were scored as such, but not included as meniscal tears in the analyses because they were present in a high percentage of the study patients (that is, $>60 \%$ ): ${ }^{27}$ further, they were equally distributed in the traumatic and non-traumatic knee and, subsequently, not related to the trauma. ${ }^{27}$ However, including such features might better explain persistent complaints. The influence of trauma with respect to the acceleration of osteoarthritis needs more studies with a longer follow-up to allow for the monitoring of complaints and functional capacity over time.

\section{Implications for practice}

In this heterogeneous population consulting after a knee injury in general practice, MRI does not seem to have additive predictive value in the 1-year prognosis after such injury. However, in larger populations, or in a more homogeneous population, MRI or physical examination might be of prognostic value; this needs further investigation. At present, only history taking shows some prognostic value and the study cannot recommend physical examination or MRI examination for prognostic use. 


\section{REFERENCES}

1. Mallen CD, Peat G, Thomas E, et al. Prognostic factors for musculoskeletal pain in primary care: a systematic review. Br J Gen Pract 2007; 57(541): 655-661.

2. Nahit ES, Hunt IM, Lunt M, et al. Effects of psychosocial and individual psychological factors on the onset of musculoskeletal pain: common and sitespecific effects. Ann Rheum Dis 2003; 62(8): 755-760.

3. Van der Waal JM, Bot SD, Terwee CB, et al. The course and prognosis of hip complaints in general practice. Ann Behav Med 2006; 31(3): 297-308.

4. Webb R, Brammah T, Lunt M, et al. Opportunities for prevention of 'clinically significant' knee pain: results from a population-based cross sectional survey. J Public Health (Oxf) 2004; 26(3): 277-284.

5. Van der Linden MW, Westert GP, de Bakker DH, et al. Tweede nationale studie naar ziekten en verrichtingen in de huisartspraktijk: klachten en aandoeningen in de bevolking en in de huisartspraktijk. [Second Dutch study for diseases and management in general practice: disorders in general practice]. Utrecht, Bilthoven: NIVEL/RIVM, 2004.

6. Boks SS, Vroegindeweij D, Koes BW, et al. Follow-up of posttraumatic ligamentous and meniscal knee lesions detected at MR imaging: systematic review. Radiology 2006; 238(3): 863-871.

7. Fujimoto E, Sumen $\mathrm{Y}$, Ochi M, et al. Spontaneous healing of acute anterior cruciate ligament (ACL) injuries - conservative treatment using an extension block soft brace without anterior stabilization. Arch Orthop Trauma Surg 2002; 122(4): 212-216.

8. Ihara H, Miwa M, Deya K, et al. MRI of anterior cruciate ligament healing. J Comput Assist Tomogr 1996; 20(2): 317-321.

9. Shelbourne KD, Jennings RW, Vahey TN. Magnetic resonance imaging of posterior cruciate ligament injuries: assessment of healing. Am J Knee Surg 1999; 12(4): 209-213.

10. Tewes DP, Fritts HM, Fields RD, et al. Chronically injured posterior cruciate ligament: magnetic resonance imaging. Clin Orthop Relat Res 1997; (335): 224-232.

11. Fithian DC, Paxton LW, Goltz DH. Fate of the anterior cruciate ligamentinjured knee. Orthop Clin North Am 2002; 33(4): 621-636, v.

12. Swirtun $L R$, Renstrom $P$. Factors affecting outcome after anterior cruciate ligament injury: a prospective study with a six-year follow-up. Scand J Med Sci Sports 2008; 18(3): 318-324

13. Thomee $P$, Wahrborg $P$, Borjesson M, et al. Self-efficacy of knee function as a pre-operative predictor of outcome 1 year after anterior cruciate ligament reconstruction. Knee Surg Sports Traumatol Arthrosc 2008; 16(2): 118-127.

14. Gillquist J, Messner K. Anterior cruciate ligament reconstruction and the long-term incidence of gonarthrosis. Sports Med 1999; 27(3): 143-156.

15. Lohmander LS, Englund PM, Dahl LL, et al. The long-term consequence of anterior cruciate ligament and meniscus injuries: osteoarthritis. Am J Sports Med 2007; 35(10): 1756-1769.

16. Roos EM, Bremander AB, Englund $M$, et al. Change in self-reported outcomes and objective physical function over 7 years in middle-aged subjects with or at high risk of knee osteoarthritis. Ann Rheum Dis 2008; 67(4): 505-510.

17. Oei EH, Nikken JJ, Verstijnen AC, et al. MR imaging of the menisci and cruciate ligaments: a systematic review. Radiology 2003; 226(3): 837-848.

18. Vincken PW, ter Braak BP, van Erkell AR, et al. Effectiveness of MR imaging in selection of patients for arthroscopy of the knee. Radiology 2002; 223(3): 739-746.

19. Kastelein M, Wagemakers HPA, Luijsterburg PAJ, et al. Assessing medial collateral ligament knee lesions in general practice. Am J Med 2008; 121(11): 982-988. e2

20. Wagemakers HP, Heintjes EM, Boks SS, et al. Diagnostic value of historytaking and physical examination for assessing meniscal tears of the knee in general practice. Clin J Sport Med 2008; 18(1): 24-30.

21. Wagemakers HPA, Luijsterburg PAJ, Boks SS, et al. Diagnostic accuracy of history taking and physical examination for assessing anterior cruciate ligament lesions of the knee in primary care. Arch Phys Med Rehabil 2010; 91(9): 1452-1459

22. Heintjes EM, Berger MY, Koes BW, et al. Knee disorders in primary care: design and patient selection of the HONEUR knee cohort. BMC Musculoskelet Disord 2005; 6: 45

23. Paice JA, Cohen FL. Validity of a verbally administered numeric rating scale to measure cancer pain intensity. Cancer Nurs 1997; 20(2): 88-93.

24. Tegner Y, Lysholm J. Rating systems in the evaluation of knee ligament injuries. Clin Orthop Relat Res 1985; (198): 43-49.

25. Ostelo RWJG, de Vet HC. Clinically important outcomes in low back pain. Best Pract Res Clin Rheumatol 2005; 19(4): 593-607.

26. Schiphof D, Boers M, Bierma-Zeinstra SM. Differences in descriptions of Kellgren and Lawrence grades of knee osteoarthritis. Ann Rheum Dis 2008; 67(7): 1034-1036

27. Boks SS, Vroegindeweij D, Koes BW, et al. Magnetic resonance imaging abnormalities in symptomatic and contralateral knees: prevalence and associations with traumatic history in general practice. Am J Sports Med 2006; 34(12): 1984-1991

28. Rasenberg El, Lemmens JA, van Kampen A, et al. Grading medial collateral ligament injury: comparison of MR imaging and instrumented valgus-varus laxity test-device. A prospective double-blind patient study. Eur J Radiol 1995; 21(1): 18-24.

29. Hoppenfield S. Physical examination of the spine and extremities. East norwalk Conn: Appleton-Century-Crofts, 1976.

30. Reider B. The Orthopaedic Physical Examination. Oxford: WB Saunders, 1999: 202-245.

31. Daniel DM. Assessing the limits of knee motion. Am J Sports Med 1991; 19(2): 139-147.

32. Torg JS, Conrad W, Kalen V. Clinical diagnosis of anterior cruciate ligament instability in the athlete. Am J Sports Med 1976; 4(2): 84-93.

33. Galway HR, Maclntosh DL. The lateral pivot shift: a symptom and sign of anterior cruciate ligament insufficiency. Clin Orthop Relat Res 1980; (147): 45-50.

34. Daniel DM. Diagnosis of knee ligament injury: tests and measurements of joint laxity. New York, NY: Churchill Livingstone, 1988: 287-300.

35. Belo JN, Berg HF, Klein Ikkink AJ, et al. NHG-Standaard Traumatische knieproblemen. Huisarts en Wetenschap 2010; 54(3): 147-158. 